\title{
IGF-1 activates the P13K/AKT signaling pathway via upregulation of secretory clusterin
}

\author{
XIUMEI MA and YONGRUI BAI \\ Department of Radiation Oncology, Renji Hospital, Shanghai Jiao Tong \\ University School of Medicine, Shanghai 200120, P.R. China
}

Received April 26, 2012; Accepted August 24, 2012

DOI: $10.3892 / \mathrm{mmr} .2012 .1110$

\begin{abstract}
Secretory clusterin (sCLU) is a type of stressinduced, pro-survival glycoprotein elevated in early-stage cancer. It enhances cancer cell survival and is associated with several types of cancer progression. In this study, we measured the PI3K/AKT signaling activity by determining the phosphorylation level of the AKT protein, namely pAKT. A549 human non-small cell lung carcinoma (NSCLC) cells were treated with insulin-like growth factor-1 (IGF-1) for various periods of time. The results showed that IGF-1 activated the PI3K/AKT signaling pathway in the A549 cells in a time-dependent manner. Western blot analysis was performed to determine the expression of sCLU protein in A549 cells treated with IGF-1. IGF-1 elevated the expression of sCLU. To determine whether SCLU is required for the IGF-1 activation of the PI3K/AKT signaling pathway, the A549 cells were treated with IGF-1 and sCLU antisense oligonuleotide (sCLU ASO). sCLU ASO blocked the IGF-1 activation of the PI3K/AKT signaling pathway. These results demonstrate that IGF-1 activates the P13K/AKT signaling pathway via the upregulation of sCLU. The present study implies that this pathway may uncover a new mechanism for cancer progression and reveal new targets for drug development in the treatment of NSCLC.
\end{abstract}

\section{Introduction}

Clusterin (CLU) is a heterodimeric, disulfide-linked 70-80 kDa glycoprotein that is induced during the regression of most, if not all, hormone-dependent epithelial tissues (1). Clusterin is expressed constitutively in many adult organs under physiological circumstances $(2,3)$. A number of studies have demonstrated that the overexpression of CLU is associated with

Correspondence to: Dr Yongrui Bai, Department of Radiation Oncology, Renji Hospital, Shanghai Jiao Tong University School of Medicine, 1630 Dongfang Road, Pudong, Shanghai 200127, P.R. China E-mail: linqmichael@gmail.com

Key words: insulin-like growth factor-1, phosphatidylinositol 3-kinase/AKT, clusterin, antisense oligonucleotide many types of cancer (4-8). However, the detailed function and regulatory mechanism of CLU remain elusive.

Clusterin expression is complex, appearing as different formations in different cell compartments. It consists of 2 isoforms: secretory clusterin (sCLU) and intracellular clusterin (nCLU). The former has been described as a long-length CLU (also known as sCLU), while the latter as a nuclear form, truncated CLU (also known as nCLU) $(9,10)$. sCLU is known as an extracellular secreted glycoprotein in the mature form that is upregulated in a variety of cell lines in response to stress and enhances cell survival (11). However, a number of studies have demonstrated that sCLU can also act as a cytoprotective protein (12-14). nCLU with nuclear localization has recently been described to be associated with cell death (15). These findings suggest that CLU is a pro-apoptotic or a survival gene. The role that CLU plays, however, remains unclear, while the difference in the function of its isoforms is also obscure.

The 3 major signaling pathways that have been identified as important in cancer are: the phosphatidylinositol 3-kinase (PI3K)/AKT signaling pathway, the protein kinase C (PKC) signaling pathway and the mitogen-activated protein kinase (MAPK)/Ras signalling pathway $(16,17)$. The PI3K/AKT signaling pathway regulates several normal cellular processes including cell proliferation, survival, growth and motility processes that are critical for tumorigenesis (17-21).

Insulin-like growth factor (IGF)-1 induces cell growth in various mammalian cell types, including several carcinoma cells (22). IGF-1 functions by binding to its receptor (IGF-1R) and IGF-1 binding proteins (IGFBPs). To date, 6 members of the IGFBP family have been described (23). The binding of IGF-1 to IGF-1R may trigger the activation of several intracellular kinases, including PI3K (24,25). Insulin receptor substrate-1 (IRS-1) is considered to be a common element in the IGF-1/PI3K signaling pathway (26).

IGF-1R, a transmembrane tyrosine kinase, is highly expressed in many cell types. Signaling through the IGF-1R is responsible for somatic growth in the fetus, whereas somatic growth in post-natal mammals is achieved through the synergistic interaction of the growth hormone and the IGFs (27). Although evidence has shown that IGF-1R may convey protumorigenic signals, its role in the development of cancer has not been elucidated (28). The inhibitors of IGF-1R, e.g., OSI-906, have been examined in clinical cancer trials to assess their clinical significance (29). As shown in a previous study, due to 
the intrinsic resistance to IGF-1R-targeted therapy, malignant tumor progression was not significanlty affected (30). Thus, in tumor therapy, a more effective target in the signaling pathway is required.

Non-small cell lung carcinoma (NSCLC) accounts for $80-85 \%$ of the total lung cancer cases and has an overall survival rate of $<15 \%$ at 5 years (31). The therapeutic options for NSCLC are limited to chemo- and radiotherapy, due to lung cancer patients with advanced stages and poor prognosis (21). Moreover, although chemotherapy is under investigation as a neoadjuvant and adjuvant strategy during the early stages of the disease and some progress has been made in the treatment of locally advanced and advanced disease, the treatment of NSCLC remains one of the great challenges of medical oncology (32). It is clear that chemotherapy has reached a plateau of activity in the treatment of NSCLC and further improvement requires the integration of novel and targeted therapies $(33,34)$.

In the present study, we measured the PI3K/ATK signaling pathway activity by determining the abundance of the pAKT protein, namely the phosphorylation of the AKT protein. Our results demonstrate that the IGF-1 activation of the PI3K/ATK signaling pathway is mediated by CLU in the A549 human NSCLC cell line. Moreover, we show that the expression of CLU is elevated by IGF-1. Thus, we believe that targeting CLU may be an alternative therapeutic strategy against malignant progression.

\section{Materials and methods}

Antibodies. Antibodies for western blot analysis were as follows: anti-CLU and anti- $\beta$-actin were purchased from Santa Cruz Biotechnology (Santa Cruz, CA, USA). Antitotal AKT and anti-phospho-AKT were from Cell Signaling Technology (Beverly, MA, USA). Horseradish peroxidase (HRP)-conjugated secondary antibodies were from Jackson ImmunoResearch (West Grove, PA, USA). OSI-906 was provided by OSI Pharmaceuticals (Melville, NY, USA).

Cell culture and treatments. The A549 human NSCLC cells, derived from human lung adenocarcinoma, were obtained from the American Type Culture Collection (Manassas, VA, USA) and maintained in Ham's F12K medium supplemented with $2 \mathrm{mM} / \mathrm{L}$-glutamine, $1.5 \mathrm{~g} / 1$ sodium bicarbonate, $10 \%$ fetal bovine serum (FBS) and $100 \mu / \mathrm{ml}$ penicillin/ streptomycin. The cells were incubated at $37^{\circ} \mathrm{C}$ with humidified air containing $5 \% \mathrm{CO}_{2}$, and passaged when confluency was reached. The cells were treated with IGF-1 for 0, 10, 30 and $60 \mathrm{~min}$ before lysis. For the OSI-906 (an IGF-1R antagonist) inhibition experiment, the cells were treated with OSI-906 at a concentration of $3 \mu \mathrm{M}$ for $30 \mathrm{~min}$. After $4 \mathrm{~h}$ of incubation, the cells were treated with IGF-1 for $30 \mathrm{~min}$.

Antisense oligonucleotide (ASO) transfection. For sCLU ASO transfection, Lipofectamine 2000, a cationic lipid (Invitrogen Inc., Carlsbad, CA, USA) was used, according to the manufacturer's instructions. Briefly, after confluency was reached, the A549 cells were lysed and seeded on 24-well plates at a concentration of $10^{5}$ cells/well. The plate was then incubated overnight. The cells were transfected with $100 \mathrm{nM}$ antisense clusterin oligonucleotide (CLU ASO) or control oligonucleotide (Oncogenex Technologies Inc., Vancouver, Canada), using $2 \mu \mathrm{l} /$ well Lipofectamine 2000. After incubating for $30 \mathrm{~min}$, the transfection medium was replaced with fresh medium. sCLU ASO was designed according to the human clusterin (NCBI: NM_203339) translation initiation site, with the sequence: 5'-CAGCAGCAGAGTCTTCATCAT-3'. Both the control and the clusterin mismatch oligonucleotide were used with the sequence: 5'- CAGCAGCAGAGTATTTATCAT-3'. The cells were lysed and used for western blot analysis 30 min later. For IGF-1 co-treatment, IGF-1 was added to the medium at a concentration of $10 \mathrm{ng} / \mathrm{ml}$. The cells were then incubated for $30 \mathrm{~min}$ before lysis.

Western blot analysis. The A549 cells were lysed directly on the culture dishes using lysis buffer $(50 \mathrm{mM}$ Tris- $\mathrm{HCl}$, $150 \mathrm{mM} \mathrm{NaCl}, 0.02 \% \mathrm{NaN}_{3}, 1 \%$ Triton X-100, $1 \mathrm{mM}$ PMSF and $1 \mu \mathrm{g} / \mathrm{ml}$ aprotinin and $1 \mu \mathrm{g} / \mathrm{ml}$ leupeptin). The protein concentration was determined using the Bradford assay kit (Bio-Rad Laboratories, Hercules, CA, USA). Cell lysates containing equal amounts of protein were separated by sodium dodecyl sulfate-polyacrylamide gel electrophoresis (SDS-PAGE) (8-10\%) and then transferred onto PVDF membranes. The membranes were initially blocked with $5 \%$ non-fat dry milk in Tris-buffered saline (TBS) for $1 \mathrm{~h}$ at room temperature and then incubated with the primary antibodies at $4^{\circ} \mathrm{C}$ overnight and HRP-conjugated secondary antibodies for $2 \mathrm{~h}$ at room temperature. Protein bands were then developed with enhanced chemiluminescence (ECL) detection reagents (Amersham Biosciences, Piscataway, NJ, USA). All analyses were carried out independently and repeated at least 3 times with similar results.

\section{Results}

Establishment of an in vitro system to detect PI3K/AKT signaling pathway. We measured the PI3K/AKT signaling activity by determining the phosphorylation level of the AKT protein, namely pAKT. The results showed that IGF-1 activated the PI3K/AKT signaling pathway in the A549 cells in a time-dependent manner (Fig. 1). In the absence of IGF-1, the pAKT protein was barely detectable in the A549 cells. A 10-min incubation time with IGF-1 was sufficient for the phosphorylation of the AKT protein. There was no notable increase in the pAKT protein level in the A549 cells incubated with IGF-1 for 30 and $60 \mathrm{~min}$. By contrast, the amount of total AKT protein showed no increase in the cells when the A549 cells were treated with IGF-1.

We further investigated whether IGF-1 activates the PI3K/ AKT signaling pathway through IGF-1R in the A549 cells. The IGF-1R antagonist, OSI-906, blocked the IGF-1-elevated phosphorylation level of the AKT protein in the A549 cells. Additionally, the ATK protein level was not affected by OSI-906 (Fig. 2).

IGF-1 upregulates sCLU protein expression. Western blot analysis was performed in order to determine the expression of sCLU protein in A549 cells treated with IGF-1. The results demonstrated that IGF-1 significantly increased sCLU protein expression (Fig. 3). 


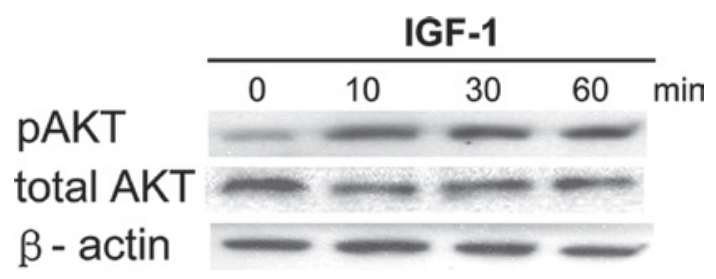

Figure 1. Western blot analysis was performed to determine the phosphorylation level of AKT and total AKT protein in A549 cells after IGF-1 treatment, at the indicated time-points $(0,10,30$ and $60 \mathrm{~min})$. $\beta$-actin and total AKT were used as the internal controls to balance the amount of loaded protein.

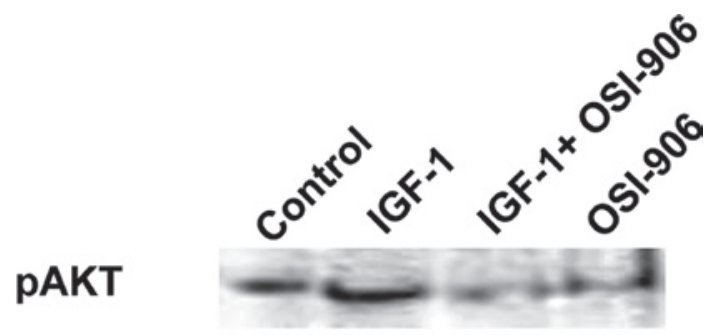

Total AKT

$\beta$ - actin

Figure 2. Using western blot analysis, we determined the phosphorylation level of AKT and total AKT protein in A549 cells treated with IGF-1, IGF-1 plus OSI-906 $(3 \mu \mathrm{M})$ or OSI-906 alone. $\beta$-actin and total AKT were used as the internal controls to balance the amount of loaded protein.

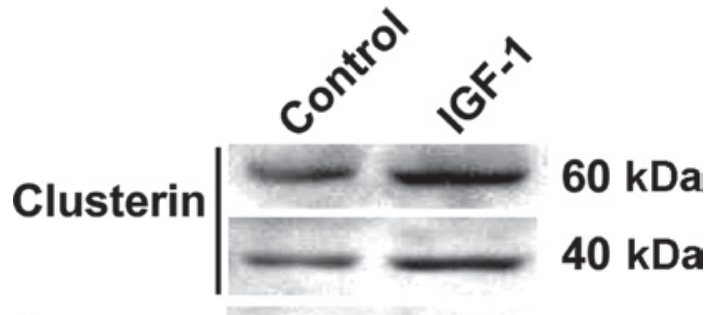

\section{$\beta$ - actin}

Figure 3. Expression of sCLU protein in A549 cells treated with IGF-1. $\beta$-actin and total AKT were used as the internal controls to balance the amount of loaded protein. The molecular weight of sCLU is shown at 40 and $60 \mathrm{kDa}$.

sCLU ASO decreases the phosphorylation level of AKT. The A549 cells were treated with sCLU ASO. The results showed that the expression of sCLU (Fig. 4A) as well as the phosphorylation level of the AKT protein in the A549 cells (Fig. 4B) were markedly inhibited.

sCLU is required for the IGF-1 stimulation of the PI3K/AKT signaling pathway. To determine whether sCLU is required for the IGF-1 activation of the PI3K/AKT signaling pathway, the A549 cells were treated with IGF-1 and sCLU ASO. The results showed that CLU ASO repressed the phosphorylation of AKT in the cells treated with IGF-1 and sCLU ASO compared to those treated with IGF-1 alone (Fig. 5).
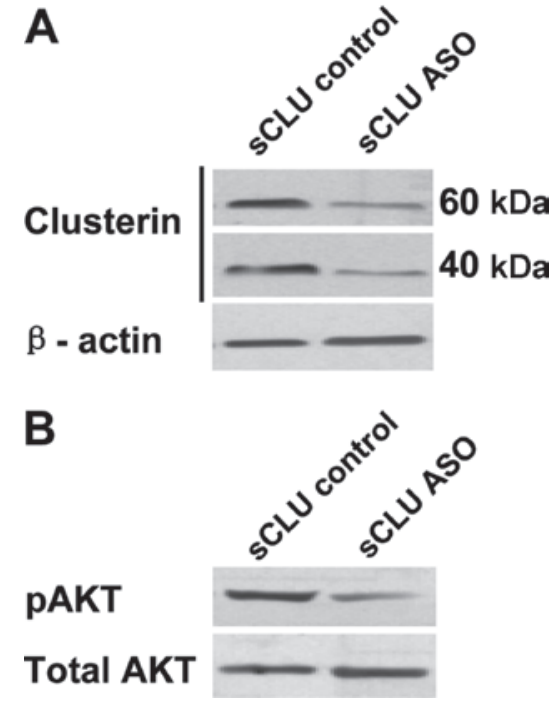

Figure 4. sCLU ASO decreased the phosphorylation level of AKT. (A) CLU ASO silenced the expression of sCLU in A549 cells. (B) sCLU ASO decreased the phosphorylation level of AKT. $\beta$-actin and total AKT were used as the internal controls to balance the amount of loaded protein.

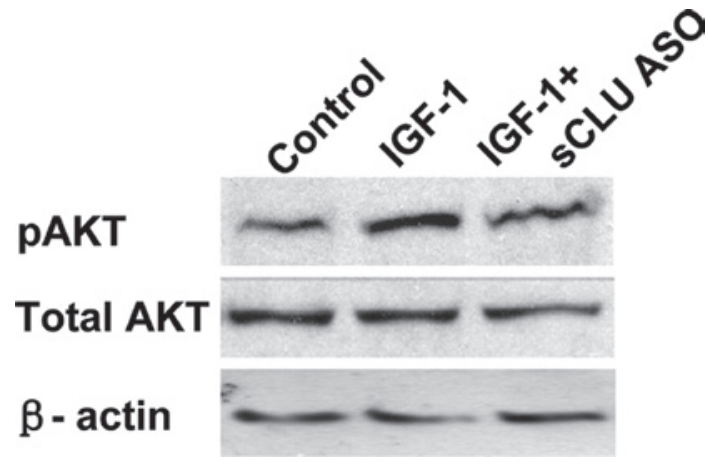

Figure 5. sCLU ASO blocked the IGF-1-activated phosphorylation of AKT $\beta$-actin and total AKT were used as the internal controls to balance the amount of loaded protein

\section{Discussion}

The PI3K/AKT pathway, in parallel to the Ras/MAPK pathway, contributes to the regulation of cell survival. These pathways are thus considered the most attractive targets for the development of novel anticancer therapies $(20,32)$. The PI3K/AKT pathway is a key regulator of cell survival through multiple downstream targets. Indeed, activated AKT, a serine/threonine kinase itself, promotes cell proliferation, growth and survival, as well as other processes involved in cancer development by phosphorylating various intracellular proteins regulating these processes (16). The IGF-1 signaling pathway is involved in cell growth and the development of cancer (35). Previous studies have demonstrated that the IGF-1 binding to its receptor, IGF-1R, activates the PI3K/AKT signaling pathway (25). These experiments, however, were all performed using normal cells. In our study, using NSCLC cells as a model of cancer, to our knowledge, we demonstrate for the first time that this activation mechanism also applies to cancer cell lines.

CLU has been described as a marker for replicative senescence, playing a protective role in inhibiting cell death (36). 
sCLU is overexpressed in many types of cancer, including human neoplasms of the prostate, breast, lung, colon and ovary $(37,38)$. Pre-clinical studies have demonstrated that clusterin antisense treatment induces apoptosis in androgendependent prostate tumor cells and delays the recurrence of androgen-independent tumors in mice (39). A recent study demonstrated that clusterin modulates the PI3K/AKT signaling pathway through the attenuation of IGF-1 during serum deprivation (40). Furthermore, several previous studies have proven that sCLU is not regulated by IGF-1 $(38,41)$. Nevertheless, whether IGF-1 upregulates sCLU via the PI3K/AKT signaling pathway remains unclear.

sCLU is vital to cancer cells, although its role remains unclear. sCLU promotes cell survival through PI3K (42). Our results revealed that sCLU is required for the PI3K/AKT signaling pathway. The stimulation of IGF-1 on the PI3K/AKT signaling pathway was significantly reduced by blocking the expression of sCLU. These results suggest that IGF-1 activates the P13K/AKT signaling pathway via the upregulation of sCLU. A previous study has shown that IGF-1 activates the PI3K/AKT signaling pathway via IRS-1 (26). In this study, we demonstrate that in NSCLC cells, sCLU plays a similar role to IRS. Thus, the association between sCLU and IRS-1 warrants further investigation.

The PI3K pathway promotes cancer cell proliferation and survival. Several studies have demonstrated that the inhibition of the PI3K/AKT signaling pathway or a downstream mediator of this pathway may provide therapeutic approaches for cancer $(43,44)$. LY294002, a PI3K inhibitor, and metformin, a 5'-adenosine monophosphate (AMP)-activated protein kinase (AMPK)-activator, have been reported to have the potential to co-enhance the inhibition of cancer growth (45). Inhibitors of proteins that are involved in the PI3K/Akt signaling pathway have yielded effective results. The overexpression of sCLU is associated with the number of cancer cells. In order to determine whether an effective inhibition of cancer growth can be achieved by targeting sCLU, additional studies are thus required.

In conclusion, the findings from our study demonstrate that IGF-1 elevates the expression of sCLU. sCLU is required for the activation of the PI3K/AKT signaling pathway. sCLU ASO represses the IGF-1-activated PI3K/AKT signaling pathway. Taken together, these results prove that IGF-1 activates the P13K/AKT signaling pathway via the upregulation of sCLU, suggesting that targeting sCLU may be an alternative therapeutic strategy against malignant progression.

\section{Acknowledgements}

The present study was funded by the National Natural Science Foundation of China (No. 31071228) and the Shanghai Municipal Natural Science Foundation (No. 09ZR1416400).

\section{References}

1. O'Sullivan J, Whyte L, Drake J and Tenniswood M: Alterations in the post-translational modification and intracellular trafficking of clusterin in MCF-7 cells during apoptosis. Cell Death Differ 10: 914-927, 2003.

2. Shannan B, Seifert M, Leskov K, et al: Challenge and promise: roles for clusterin in pathogenesis, progression and therapy of cancer. Cell Death Differ 13: 12-19, 2006.
3. Tenniswood MP, Guenette RS, Lakins J, Mooibroek M, Wong P and Welsh JE: Active cell death in hormone-dependent tissues. Cancer Metastasis Rev 11: 197-220, 1992.

4. Redondo M, Villar E, Torres-Munoz J, Tellez T, Morell M and Petito CK: Overexpression of clusterin in human breast carcinoma. Am J Pathol 157: 393-399, 2000.

5. Miyake H, Gleave M, Kamidono S and Hara I: Overexpression of clusterin in transitional cell carcinoma of the bladder is related to disease progression and recurrence. Urology 59: 150-154, 2002.

6. Xie D, Lau SH, Sham JS, et al: Up-regulated expression of cytoplasmic clusterin in human ovarian carcinoma. Cancer 103: 277-283, 2005.

7. Kang YK, Hong SW, Lee $\mathrm{H}$ and Kim WH: Overexpression of clusterin in human hepatocellular carcinoma. Hum Pathol 35: 1340-1346, 2004.

8. Miyake H, Hara S, Arakawa S, Kamidono S and Hara I: Overexpression of clusterin is an independent prognostic factor for nonpapillary renal cell carcinoma. J Urol 167: 703-706, 2002.

9. Leskov KS, Klokov DY, Li J, Kinsella TJ and Boothman DA: Synthesis and functional analyses of nuclear clusterin, a cell death protein. J Biol Chem 278: 11590-11600, 2003.

10. Moretti RM, Marelli MM, Mai S, et al: Clusterin isoforms differentially affect growth and motility of prostate cells: possible implications in prostate tumorigenesis. Cancer Res 67: 10325-10333, 2007.

11. Tenniswood M, Flanagan L, Whyte L and Chatterjee N: Effects of clusterin over-expression on metastatic progression and therapy in breast cancer. BMC Cancer 10: 107, 2010.

12. Wu AJ, Park, II, Zhaung L and Lee C: Response to a lethal dose of heat shock by a transient up-regulation of clusterin expression followed by down-regulation and apoptosis in prostate and bladder cancer cells. Prostate 53: 277-285, 2002.

13. Sensibar JA, Sutkowski DM, Raffo A, et al: Prevention of cell death induced by tumor necrosis factor alpha in LNCaP cells by overexpression of sulfated glycoprotein-2 (clusterin). Cancer Res 55: 2431-2437, 1995

14. Miyake H, Nelson C, Rennie PS and Gleave ME: Testosteronerepressed prostate message- 2 is an antiapoptotic gene involved in progression to androgen independence in prostate cancer. Cancer Res 60: 170-176, 2000.

15. Scaltriti M, Santamaria A, Paciucci R and Bettuzzi S: Intracellular clusterin induces $\mathrm{G} 2-\mathrm{M}$ phase arrest and cell death in PC-3 prostate cancer cells1. Cancer Res 64: 6174-6182, 2004.

16. Faivre S, Kroemer G and Raymond E: Current development of mTOR inhibitors as anticancer agents. Nat Rev Drug Discov 5: 671-688, 2006.

17. Engelman JA: Targeting PI3K signalling in cancer: opportunities, challenges and limitations. Nat Rev Cancer 9: 550-562, 2009.

18. Downward J: Targeting RAS and PI3K in lung cancer. Nat Med 14: 1315-1316, 2008.

19. Gustafson AM, Soldi R, Anderlind C, et al: Airway PI3K pathway activation is an early and reversible event in lung cancer development. Sci Transl Med 2: 26ra25, 2010.

20. Luo J, Manning BD and Cantley LC: Targeting the PI3K-Akt pathway in human cancer: rationale and promise. Cancer Cell 4: 257-262, 2003.

21. Wang J, Zhang N, Li B, et al: Decline of serum CYFRA21-1 during chemoradiotherapy of NSCLC: a probable predictive factor for tumor response. Tumour Biol 32: 689-695, 2011.

22. Resnicoff M, Ambrose D, Coppola D and Rubin R: Insulin-like growth factor-1 and its receptor mediate the autocrine proliferation of human ovarian carcinoma cell lines. Lab Invest 69: 756-760, 1993.

23. Schneider MR, Lahm H, Wu M, Hoeflich A and Wolf E: Transgenic mouse models for studying the functions of insulinlike growth factor-binding proteins. FASEB J 14: 629-640, 2000.

24. LeRoith D, Werner H, Beitner-Johnson D and Roberts CT Jr: Molecular and cellular aspects of the insulin-like growth factor I receptor. Endocr Rev 16: 143-163, 1995.

25. Saltiel AR and Kahn CR: Insulin signalling and the regulation of glucose and lipid metabolism. Nature 414: 799-806, 2001.

26. Myers MG Jr, Sun XJ, Cheatham B, et al: IRS-1 is a common element in insulin and insulin-like growth factor-I signaling to the phosphatidylinositol 3'-kinase. Endocrinology 132: 1421-1430, 1993

27. Adams TE, Epa VC, Garrett TP and Ward CW: Structure and function of the type 1 insulin-like growth factor receptor. Cell Mol Life Sci 57: 1050-1093, 2000. 
28. Baserga R, Peruzzi F and Reiss K: The IGF-1 receptor in cancer biology. International journal of cancer. Int J Cancer 107: 873-877, 2003

29. Gualberto A and Pollak M: Emerging role of insulin-like growth factor receptor inhibitors in oncology: early clinical trial results and future directions. Oncogene 28: 3009-3021, 2009.

30. Ulanet DB, Ludwig DL, Kahn CR and Hanahan D: Insulin receptor functionally enhances multistage tumor progression and conveys intrinsic resistance to IGF-1R targeted therapy. Proc Natl Acad Sci USA 107: 10791-10798, 2010.

31. Ko JC, Hong JH, Wang LH, et al: Role of repair protein Rad51 in regulating the response to gefitinib in human non-small cell lung cancer cells. Mol Cancer Ther 7: 3632-3641, 2008.

32. Gridelli C, Rossi A and Maione P: Treatment of non-small-cell lung cancer: state of the art and development of new biologic agents. Oncogene 22: 6629-6638, 2003.

33. Pallis AG, Serfass L, Dziadziusko R, et al: Targeted therapies in the treatment of advanced/metastatic NSCLC. Eur J Cancer 45 2473-2487, 2009.

34. Carney DN: Lung cancer--time to move on from chemotherapy. N Engl J Med 346: 126-128, 2002.

35. Baserga R: The IGF-I receptor in cancer research. Exp Cell Res 253: 1-6, 1999

36. Petropoulou C, Trougakos IP, Kolettas E, Toussaint $\mathrm{O}$ and Gonos ES: Clusterin/apolipoprotein $\mathrm{J}$ is a novel biomarker of cellular senescence that does not affect the proliferative capacity of human diploid fibroblasts. FEBS Lett 509: 287-297, 2001.

37. Stahel RA and Zangemeister-Wittke U: Antisense oligonucleotides for cancer therapy-an overview. Lung Cancer 41 Suppl 1 S81-S888, 2003.

38. Suzuki M, C. Goetz E, Veneziano G, et al: Secretory clusterin (sCLU) is a hallmark sensor of DNA damage, cell stress, and cellular senescence: Evidence for similar regulation of sCLU expression after cellular stress and replicative senescence. ICS 1299: 150-157, 2007.
39. Zellweger T, Miyake H, July LV, Akbari M, Kiyama S and Gleave ME: Chemosensitization of human renal cell cancer using antisense oligonucleotides targeting the antiapoptotic gene clusterin. Neoplasia 3: 360-367, 2001.

40. Jo H, Jia Y, Subramanian KK, Hattori H and Luo HR: Cancer cell-derived clusterin modulates the phosphatidylinositol 3'-kinase-Akt pathway through attenuation of insulin-like growth factor 1 during serum deprivation. Mol Cell Biol 28: 4285-4299, 2008.

41. Goetz EM, Shankar B, Zou Y, et al: ATM-dependent IGF-1 induction regulates secretory clusterin expression after DNA damage and in genetic instability. Oncogene 30: 3745-3754, 2011.

42. Ammar $\mathrm{H}$ and Closset JL: Clusterin activates survival through the phosphatidylinositol 3-kinase/Akt pathway. J Biol Chem 283: 12851-12861, 2008.

43. Rychahou PG, Jackson LN, Silva SR, Rajaraman S and Evers BM: Targeted molecular therapy of the PI3K pathway: therapeutic significance of PI3K subunit targeting in colorectal carcinoma. Ann Surg 243: 833-842; discussion 843-834, 2006.

44. Pitt SC, Chen $\mathrm{H}$ and Kunnimalaiyaan M: Inhibition of phosphatidylinositol 3-kinase/Akt signaling suppresses tumor cell proliferation and neuroendocrine marker expression in GI carcinoid tumors. Ann Surg Oncol 16: 2936-2942, 2009.

45. Li C, Liu VW, Chan DW, Yao KM and Ngan HY: LY294002 and metformin cooperatively enhance the inhibition of growth and the induction of apoptosis of ovarian cancer cells. Int J Gynecol Cancer 22: 15-22, 2012 . 